\title{
A study on the machinability of some metal alloys using grey TOPSIS method
}

\author{
Swarat Dey and Shankar Chakraborty*
}

Department of Production Engineering, Jadavpur University, Kolkata - 700 032, West Bengal, India

\begin{tabular}{l}
\hline C H R O N I C L E \\
\hline Article history: \\
Received June 25, 2015 \\
Received in revised format: \\
September 2, 2015 \\
Accepted September 4, 2015 \\
Available online \\
September 5 2015 \\
\hline Keywords: \\
Machinability \\
Alloys \\
Aluminium \\
Copper \\
Alloy steel \\
Grey theory \\
TOPSIS
\end{tabular}

\section{Introduction}

The machinability of a material can be defined as the ease with which it can be cut (machined) by a tool permitting material removal with a satisfactory finish at the lowest possible cost. Materials with good machinability require less power to cut, can be cut quickly, and easily obtain a good finish without wearing the tooling much. Practically, no two materials subject to machining can behave alike when cutting them with the same tool, at the same cutting speed and feed rate, using the same machine and working under similar conditions. Some may produce long curly chips (like mild steel), some may produce short chips (like cast iron), some may get a smooth finish, some may end up with a rough surface, some may produce chatter, and some may produce lots of heat and quickly blunt the tool. The machinability is not a specific property of a material, but a mode of its behavior during cutting, and assessments of machinability should, therefore, specify the general conditions of cutting. Machinability

\footnotetext{
* Corresponding author.

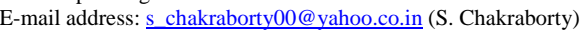


is related to all aspects of a manufacturing process, such as product design, quality control, and especially, process planning and machining operations. Thus, it is an important consideration for engineers in materials selection, and also is the base of selecting cutting tools and optimizing the machining parameters. The machinability aspect is of considerable interest to the production engineers to study the machinability of a work material in advance so that the processing can be planned efficiently. Machinability of a material is one of the prime metal cutting parameters that has influence in selecting several other cutting parameters.

A number of variables, like inherent properties or characteristics of work materials, tool geometry, cutting tool material, nature of tool engagement with the workpiece, cutting conditions, cutting fluid, type of cutting, and machine tool rigidity and its capacity greatly influence machinability of materials. Other dependent process variables, such as tool life, specific power consumption, cutting forces, achievable surface finish, dimensional accuracy, temperature generated, noise, vibration and chip characteristics are also directly related to machinability (Rao, 2006). Depending on the technical and economic needs of a machining process, some criteria may have primary or secondary roles in machinability evaluation. It is observed that addition of a certain proportion of an alloying material in the base metal can significantly improve its machinability characteristics. Like, in iron and steel, presence of sulphur (up to $0.35 \%$ ) helps in breaking of chips while improving machinability. An alloy may be described as a metal with more than one element, the other elements are being added to enhance the base metal's properties to suit a particular application. In order to fulfill the continuous use of various alloys in diverse field of engineering, it becomes quite important to study their machinability characteristics so as to guide the production engineers in selecting the proper cutting conditions and the related parameters. Taking a typical free cutting steel (AISI B1112, a steel with a chemical composition C 0.08-0.13\%, Mn 0.60-0.90\%, P 0.09-0.13\% and S 0.16-0.23\%, and having a hardness of $160 \mathrm{BHN}$ ) as a reference material and allocating a machinability rating (also referred as machinability index) of 100 to it, the machinability ratings can be allocated to other materials (Mills, 1983).

With the increasing number of new materials available in the market every year, the manufacturers are facing great difficulties in selecting the most appropriate material for their products. Thus, there is always an ardent need to adopt a simple systematic methodology for efficient and effective evaluation of machinability of various work materials. In this paper, a multi-criteria decision-making (MCDM) tool in the form of grey TOPSIS (technique for order preference by similarity to ideal solution) method is applied to study the machinability characteristics of three types of alloys, i.e. aluminium alloys, copper alloys and alloy steels. Various important mechanical properties of those alloys are taken into consideration while identifying the best machinable alloys from the list of candidate alternatives. As it is often difficult to express the mechanical properties of the considered alloys using single numbers, they are expressed in terms of grey numbers. The TOPSIS method, being an easily comprehensible MCDM technique and having a strong mathematical background, is also quite suitable to this type of evaluation and selection problem. The grey TOPSIS method thus helps the engineers to evaluate the machinability charactertics of the considered metal alloys for a given machining application and selection of a proper alloy to strengthen the present work material selection procedure.

\section{Literature review}

Enache et al. (1995) presented a new mathematical model using some of the evaluation criteria influencing a material's machinability property with the determination of partial and global machinability indices. Kim et al. (2002) developed a cutting speed optimization program to study and enhance the machining precision and tool life in high speed machining operation using ball end mill. The machining precision and tool life were compared in free surface machining conducted by general cutting method and the technique of optimal cutting speed. Rao and Gandhi (2002) developed a methodology to determine the machinability of work materials for a specific machining operation using digraph and matrix approaches. Boubekri et al. (2003) proposed a methodology for machinability 
evaluation of steels while directly using the cutting force and surface roughness data, along with the subsequent development of an aggregate machinability indicator. Manna and Bhattacharayya (2003) presented the results of an experimental investigation on the machinability study of silicon carbide particulate aluminium metal matrix composite (Al/SiC-MMC) during turning using fixed rhombic tools. It was claimed that the research findings would provide useful machining solution by utilizing fixed rhombic tooling during processing of $\mathrm{Al} / \mathrm{SiC}-\mathrm{MMC}$.

Rech et al. (2004) developed a novel approach to compare the machinability of three mold steels during rough milling. Davim and Reis (2004) studied the influences of various cutting parameters, like cutting velocity, feed rate etc. on power, specific cutting pressure, surface roughness and International dimensional precision in polyetheretherketone (PEEK) reinforced with 30\% of glass fibre (PEEK GF30) material. Davim and Mata (2005) studied the machinability in turning processes of fiber reinforced plastics using polycrystalline diamond cutting tools. Orthogonal arrays and analysis of variance were employed to investigate the influence of cutting parameters on specific cutting pressure and surface roughness. Stoić et al. (2005) tested the machinability of hard materials in high speed turning process and also investigated the influence of cutting parameters on machinability rates.

Šalak et al. (2006) studied the machinability of powder metallurgy (PM) steels using different methods for various cutting processes. It was shown that face turning test method was simple and could fulfill some of the major requirements while assessing the machinability of PM steels in turning. Şeker and Hasirci (2006) presented the results of machining tests as conducted to study the effect of microstructure and mechanical properties of austempered ductile irons on cutting forces and surface roughness. Rao (2006) presented a systematical procedure to evaluate the machinability of work materials for a given machining operation. Davim and Figueira (2007) employed orthogonal array and analysis of variance to study the machinability property of cold work tool steel. Morehead et al. (2007) investigated the machinability of equal channel angular extrusion (ECAE)-processed pure copper using both tungsten carbide and polycrystalline diamond cutting tools to facilitate broader applications of ECAE-processed ultrafine-grained copper.

Hoseiny et al. (2012) compared machinability of some of the most popular grades of pre-hardened plastic mold steel in two milling and two drilling operations. Sridharan and Muthukrishnan (2013) presented a comparison of machinability of jute fiber reinforced composites prepared using untreated and alkali treated fiber. Xu et al. (2013) developed a polar diagram method for describing and evaluating the potential machinability properties of different workpiece materials. Lobato et al. (2014) applied bio-inspired optimization methods to study the machinability of stainless steel AISI 420 while considering tool life and cutting force in terms of cutting speed, feed per tooth and axial depth of cut in an end milling process. Sameer Kumar and Suman (2014) selected magnesium alloy materials for use in automotive wheel applications using MCDM methods. Wang et al. (2014) proposed a new method to evaluate the machinability of difficult-to-cut materials and then evaluated the machinability of four kinds of superalloys.

\section{Grey TOPSIS method}

Grey number has been originated from the concept of grey theory (Deng, 1982; 1989) which can suitably consider insufficient and incomplete information. Grey theory is a popular method used to study behavior of mathematical systems having uncertain information. It is observed that information derived from real world is always uncertain or incomplete. Hence, extension of the applications of grey numbers from white numbers (crisp values) is often necessary for real world applications. The grey number is a number with uncertain/incomplete information. In grey theory, if the system information is fully known, the system is called a white system. On the other hand, if the information is not known at all, it is a black system. A system with partially known information is called a grey system. The advantage of grey theory over fuzzy theory is that it can consider condition of fuzziness, which simply 
states that it can deal flexibly with fuzzy situation. Thus, white number, grey number and black number are the three classifications to differentiate the level of uncertainty in information (Li et al., 2007; Lin et al., 2008; Zavadskas et al., 2009).

Let $\otimes G=[\underline{G}, \bar{G}]=\{G \mid \underline{G} \leq G \leq \bar{G}, \underline{G}$ and $\bar{G} \in R\}$. Then, $\otimes G$ which contains two real numbers $\underline{G}$ (the lower limit of $\otimes G$ ) and $\bar{G}$ (the upper limit of $\otimes G$ ) is defined as below:

a) If $\underline{G} \rightarrow-\infty$ and $\bar{G} \rightarrow \infty$, then $\otimes G$ is called the black number which means without any meaningful information.

b) Else if $\underline{G}=\bar{G}$, then $\otimes G$ is called the white number which means with complete information.

c) Otherwise, $\otimes G=[\underline{G}, \bar{G}]$; $\otimes G$ is called the grey number with insufficient and uncertain information.

Let there are two sets of grey numbers denoted by $\otimes G_{1}=\left[\underline{G}_{1}, \bar{G}_{1}\right]$ and $\otimes G_{2}=\left[\underline{G}_{2}, \bar{G}_{2}\right]$. The basic mathematical operations of these two sets of grey numbers can be given as follows (Turskis \& Zavadskas, 2010):

$$
\begin{aligned}
& \otimes G_{1}+\otimes G_{2}=\left[\underline{G}_{1}+\underline{G}_{2}, \bar{G}_{1}+\bar{G}_{2}\right] \\
& \otimes G_{1}-\otimes G_{2}=\left[\underline{G}_{1}-\underline{G}_{2}, \bar{G}_{1}-\bar{G}_{2}\right] \\
& \otimes G_{1} \times \otimes G_{2}=\left[\min \left(\underline{G}_{1} \underline{G}_{2}, \underline{G}_{1} \bar{G}_{2}, \bar{G}_{1} \underline{G}_{2}, \bar{G}_{1} \bar{G}_{2}\right), \max \left(\underline{G}_{1} \underline{G}_{2}, \underline{G}_{1} \bar{G}_{2}, \bar{G}_{1} \underline{G}_{2}, \bar{G}_{1} \bar{G}_{2}\right)\right] \\
& \otimes G_{1} \div \otimes G_{2}=\left[\underline{G}_{1}, \bar{G}_{1}\right] \times\left[\frac{1}{\underline{G}_{2}}, \frac{1}{\bar{G}_{2}}\right]
\end{aligned}
$$

In this paper, TOPSIS method is applied to study the machinability characteristics of some of the alloys of three metals where different mechanical properties (criteria) of the alloys are expressed in grey numbers. This method is also quite suitable for solving group decision-making problems in an uncertain environment. The procedural steps of grey TOPSIS method are demonstrated as follows (Jadidi et al. 2008; Zolfani \& Antucheviciene, 2012; Sadeghi et al. 2013):

Step 1: Develop the grey decision matrix.

$$
D=\left[\begin{array}{cccc}
\otimes G_{11} & \otimes G_{12} & \ldots & \otimes G_{1 n} \\
\otimes G_{21} & \otimes G_{22} & \ldots & \otimes G_{2 n} \\
\ldots & \ldots & \ldots & \ldots \\
\otimes G_{m 1} & \otimes G_{m 2} & \ldots & \otimes G_{m n}
\end{array}\right]
$$

where $\otimes G_{i j}$ is the performance of $i^{\text {th }}$ alternative with respect to $j^{\text {th }}$ criterion expressed in grey numbers.

Step 2: Normalize the grey decision matrix.

$$
D^{*}=\left[\begin{array}{cccc}
\otimes G_{11}^{*} & \otimes G_{12}^{*} & \ldots & \otimes G_{1 n}^{*} \\
\otimes G_{21}^{*} & \otimes G_{22}^{*} & \ldots & \otimes G_{2 n}^{*} \\
\ldots & \ldots & \ldots & \ldots \\
\otimes G_{m 1}^{*} & \otimes G_{m 2}^{*} & \ldots & \otimes G_{m n}^{*}
\end{array}\right]
$$

where $\otimes G_{i j}^{*}=\left(\frac{\underline{G}_{i j}}{\frac{1}{2}\left[\sum_{i=1}^{m} \bar{G}_{i j}+\sum_{i=1}^{m} \underline{G}_{i j}\right]}, \frac{\overline{G_{i j}}}{\frac{1}{2}\left[\sum_{i=1}^{m} \bar{G}_{i j}+\sum_{i=1}^{m} \underline{G}_{i j}\right]}\right)$ 
Step 3: Determine the criteria weights.

In a group decision-making environment, say, there are $k$ persons and the weight of $j^{\text {th }}$ criterion can be calculated using the following equation:

$$
\otimes w_{j}=\left(\otimes w_{1 j}^{p_{1}} \cdot \otimes w_{2 j}^{p_{2}} \ldots . \otimes w_{l j}^{p_{l}}\right)^{\frac{1}{\sum p_{l}}}
$$

where $\otimes w_{l j}^{p_{k}}(j=1,2, \ldots, n)$ is the weight which the $l^{\text {th }}$ decision maker $(l=1,2, \ldots, k)$ assigns to $j^{\text {th }}$ criterion and is described by grey number $\otimes w_{l j}=\left[\underline{w}_{l j}, \bar{w}_{l j}\right]$.

Step 4: Develop the weighted normalized grey decision matrix

$$
V=\left[\begin{array}{cccc}
\otimes V_{11} & \otimes V_{12} & \ldots & \otimes V_{1 n} \\
\otimes V_{21} & \otimes V_{22} & \ldots & \otimes V_{2 n} \\
\ldots & \ldots & \ldots & \ldots \\
\otimes V_{m 1} & \otimes V_{m 2} & \ldots & \otimes V_{m n}
\end{array}\right]
$$

where $\otimes V_{i j}=\otimes G_{i j}^{*} \times \otimes w_{j}$.

Step 5: Determine the positive and the negative ideal solutions.

For $m$ possible alternatives set $A=\left\{A_{1}, A_{2}, \ldots, A_{m}\right\}$, the positive ideal solution $A^{\max }=$ $\left\{\otimes G_{1}^{\max }, \otimes G_{2}^{\max }, \ldots, \otimes G_{n}^{\max }\right\}$ can be obtained as below:

$$
A^{\max }=\left\{\left[\max \underline{V}_{i 1}, \max \bar{V}_{i 1}\right]\left[\max \underline{V}_{i 2}, \max \bar{V}_{i 2}\right], \ldots,\left[\max \underline{V}_{i n}, \max \bar{V}_{i n}\right]\right\}(1 \leq i \leq m)
$$

On the other hand, the negative solution $A^{\mathrm{min}}=\left\{\otimes G_{1}^{\min }, \otimes G_{2}^{\min }, \ldots, \otimes G_{n}^{\min }\right\}$ can be derived as follows:

$$
A^{\min }=\left\{\left[\min \underline{V}_{i 1}, \min \bar{V}_{i 1}\right],\left[\min \underline{V}_{i 2}, \min \bar{V}_{i 2}\right], \ldots,\left[\min \underline{V}_{i n}, \min \bar{V}_{i n}\right]\right\}(1 \leq i \leq m)
$$

Step 6: Calculate the separation distances from the positive ideal and the negative ideal solutions.

The separation distance from the positive ideal solution is computed as follows:

$$
S_{i j}^{\max }=\left(\bar{V}_{i j}-\bar{A}_{\max }\right)^{2}+\left(\underline{V}_{i j}-\underline{A}_{\max }\right)^{2}
$$

The separation distance from the negative ideal solution is calculated as below:

$$
S_{i j}^{\min }=\left(\bar{V}_{i j}-\bar{A}_{\min }\right)^{2}+\left(\underline{V}_{i j}-\underline{A}_{\min }\right)^{2}
$$

Step 7: Compute $T^{\mathrm{max}}$ and $T^{\mathrm{min}}$ values.

$$
T^{\max }=\sum_{j=1}^{n} S_{i j}^{\max }
$$


$T^{\min }=\sum_{j=1}^{n} S_{i j}^{\min }$

Step 8: Calculate the separation measures $\left(D^{+}\right.$and $\left.D^{-}\right)$.

$$
\begin{array}{ll}
D^{+}=\sqrt{\frac{T^{\max }}{2}} & \text { for } 1 \leq i \leq m \\
D^{-}=\sqrt{\frac{T^{\min }}{2}} & \text { for } 1 \leq i \leq m
\end{array}
$$

Step 9: Compute the relative closeness index for each alternative.

$$
C=\frac{D^{-}}{D^{+}+D^{-}}
$$

The alternatives are now ranked depending on their relative closeness index values. The higher the closeness index, the better is the alternative.

\section{Illustrative examples}

In order to demonstrate the applicability of grey TOPSIS method in identifying the best machinable alloys from a group of candidate alternatives, the following three examples are illustrated.

\subsection{Aluminium alloys}

Aluminium, with a density of $2700 \mathrm{~kg} / \mathrm{m}^{3}$, is the lightest amongst all ordinary metals, approximately three times as light as steel. Along with the other metals, aluminium alloys are widely used in many customary processes, like machining, forming, bending, vessel-making and stamping. The absolute requirement for light structures makes aluminium and its alloys to take a major share as a suitable material in sky. In aeronautical applications, precision casting of aluminum components has found considerable attention due to reduced cost of the components. While using aluminium alloys, the design of high-speed ships is modified, by reducing the weight of hulls by $40 \%$ to $50 \%$ over steel. High corrosion resistance, even in water, makes aluminium and its alloys most suitable for more durable hulls, masts and superstructures on boats and bridges. These favorable physical properties of aluminium and its alloys are also responsible for their growing use in mechanical applications. Machines having moving parts, such as robots, are being made with an increasing number of aluminium components to reduce inertia. With respect to heat exchange (liquid-to-liquid or liquid-to-gas), aluminium's thermal conductivity plays an important role in electronics, seawater desalination, HVAC exchangers and plastics industry, where aluminium alloy molds with enhanced mechanical properties are widely used to shorten fabrication cycles by up to $30 \%$. In the near past, newer aluminum casting processes have been developed for reduced manufacturing costs and the properties of aluminum cast alloys are optimized to increase tensile ductility and fracture toughness, without any adverse effect on tensile and yield strength.

In this paper, the machinability characteristics of three series of cast aluminium alloys, i.e. A357, A224 and 7475 are studied using grey TOPSIS method. The chemical compositions of the considered aluminium alloys are as follows: for A357 series: Si 7\%, Mg 0.6\%, Ti 0.15\%, $\mathrm{Cu}<0.2 \%, \mathrm{Fe}<0.2 \%$, $\mathrm{Mn}<0.1 \%$, $\mathrm{Zn}<0.1 \%$, others < 0.15\%, Al rest; for A224 series: $\mathrm{Si}<0.067 \%$, Ti $0.35 \%$, Cu 5\%, Mn $0.35 \%$, Zr 0.2\%, others $0.1 \%$, Al rest; and for 7475 series: Mg 2.4\%, Ti 0.1\%, Cu 1.8\%, Zn 5.7\%, 
others $0.1 \%$, Al rest. The machinability characteristics of eight such aluminium alloys from these three series are evaluated with respect to five mechanical properties, i.e. yield strength (in MPa), tensile strength (in $\mathrm{MPa}$ ), elongation at fracture (in \%), strain energy density $\left(\mathrm{MJ} / \mathrm{m}^{3}\right)$ and quality evaluation index (in MPa). Among these, yield strength, tensile strength and quality evaluation index require higher values (beneficial criteria). On the other hand, minimum values are required for elongation at fracture and strain energy density (non-beneficial criteria). The corresponding decision matrix for the machinability study of aluminium alloys is developed in Table 1 . In this table, the acronyms F and R associated with the alloy designations stand for flat and round respectively. On the other hand, the acronyms S and C denote Sophia and conventional casting processes respectively.

The maximum stress a metal alloy can withstand before failing is its ultimate tensile strength. A yield strength or yield point of a metal alloy is defined as the stress at which it begins to deform plastically. Elongation at fracture is defined as the percentage increase in length to initial length before fracture. The strain energy density of a metal alloy is defined as its strain energy per unit volume. It is equal to the area under the stress-strain diagram of a metal alloy. Quality index (QI) is a measure of machinability which can be expressed as QI $=\mathrm{TS}+\log _{10} \mathrm{EF}$ where TS is the tensile strength and EF is the elongation at fracture (Alexopoulos \& Pantelakis, 2004; Alexopoulos, 2007). These mechanical properties of aluminium alloys are first converted into their corresponding grey numbers considering $1 \%$ greyness in calculation. This grey decision matrix with $1 \%$ greyness is exhibited in Table 2 which is subsequently normalized using Eq. (6). In order to develop the weighted normalized grey decision matrix, the grey weights of the considered mechanical properties (criteria) of aluminium alloys need to be calculated based on Eq. (7). The grey scale for the criteria weights is shown in Table 3 and the grey weights for five criteria of aluminium alloys considering a group decision-making environment involving four decision makers are given in Table 4. Now, using the grey data of Table 2 and grey weights of Table 4, the related weighted normalized grey decision matrix is developed in Table 5. Employing Eqs. (9)-(17), the relative closeness value for each candidate aluminium alloy is then computed, as shown in Table 6.

It is observed from Table 6 that aluminium alloy A357RC ranks first from the machinability point of view. Although it has moderate yield strength and tensile strength, but its minimum elongation at fracture and strain energy density drive it to attain the top position in the entire ranking list. Aluminium alloy 7475FS is the most difficult to machine due to its higher yield strength and tensile strength. In Table 7, the effects of greyness in the mechanical properties of aluminium alloys on the ranking performance of the employed grey TOPSIS method are exhibited. It becomes clear from this table that the rankings of the candidate aluminium alloys remain unaltered with the changing greyness values.

\section{Table 1}

Mechanical properties of aluminium alloys

\begin{tabular}{lccccc}
\hline $\begin{array}{l}\text { Aluminium } \\
\text { alloy }\end{array}$ & $\begin{array}{c}\text { Yield strength } \\
(\text { YS })\end{array}$ & $\begin{array}{c}\text { Tensile strength } \\
(\mathrm{TS})\end{array}$ & $\begin{array}{c}\text { Elongation at } \\
\text { fracture (EF) }\end{array}$ & $\begin{array}{c}\text { Strain energy } \\
\text { density (SED) }\end{array}$ & Quality index (QI) \\
\hline A357FS & 303 & 372 & 12.19 & 46.04 & 535.0 \\
A357RS & 305 & 362 & 7.92 & 29.36 & 497.0 \\
A357FC & 305 & 340 & 2.16 & 8.08 & 389.9 \\
A357RC & 289 & 319 & 1.37 & 4.87 & 339.5 \\
A224FS & 257 & 387 & 7.85 & 30.71 & 521.1 \\
A224RS & 236 & 369 & 8.96 & 33.41 & 511.6 \\
7475FS & 479 & 506 & 4.92 & 27.99 & 609.8 \\
7475RS & 465 & 491 & 2.61 & 14.43 & 553.7 \\
\hline
\end{tabular}


Table 2

Grey decision matrix for aluminium alloys

\begin{tabular}{lccccc}
\hline Aluminium alloy & YS & TS & EF & SED & QI \\
\hline A357FS & $(299.97,306.03)$ & $(368.28,357.72)$ & $(12.09,12.31)$ & $(45.58,46.50)$ & $(529.65,540.35)$ \\
A357RS & $(301.95,308.05)$ & $(358.38,365.62)$ & $(7.84,8.00)$ & $(29.07,29.65)$ & $(492.03,501.97)$ \\
A357FC & $(301.95,308.05)$ & $(336.6,343.4)$ & $(2.14,2.18)$ & $(8.00,8.16)$ & $(386.00,393.80)$ \\
A357RC & $(286.11,291.89)$ & $(315.81,322.19)$ & $(1.36,1.38)$ & $(4.82,4.92)$ & $(336.10,342.90)$ \\
A224FS & $(254.43,259.57)$ & $(383.13,390.87)$ & $(7.77,7.93)$ & $(30.40,31.02)$ & $(515.90,526.31)$ \\
A224RS & $(233.64,238.36)$ & $(365.31,372.69)$ & $(8.87,9.05)$ & $(33.07,33.74)$ & $(506.48,516.72)$ \\
7475FS & $(474.21,483.79)$ & $(500.94,511.06)$ & $(4.87,4.97)$ & $(27.71,28.27)$ & $(603.70,615.90)$ \\
7475RS & $(460.35,469.65)$ & $(486.09,495.91)$ & $(2.58,2.64)$ & $(14.28,14.57)$ & $(548.16,559.24)$ \\
\hline
\end{tabular}

Table 3

Scale for criteria weights

\begin{tabular}{ll}
\hline Scale & $\otimes w$ \\
\hline Very low & $(0.1,0.2)$ \\
Low & $(0.2,0.3)$ \\
Medium low & $(0.3,0.4)$ \\
Medium & $(0.4,0.5)$ \\
Medium high & $(0.5,0.6)$ \\
High & $(0.6,0.7)$ \\
Very high & $(0.7,0.8)$ \\
\hline
\end{tabular}

Table 4

Grey weights for mechanical properties of aluminium alloys

\begin{tabular}{lccccc}
\hline Criteria & YS & TS & EF & SED & QI \\
\hline Grey weight & $(0.648,0.748)$ & $(0.573,0.674)$ & $(0.245,0.346)$ & $(0.346,0.447)$ & $(0.141,0.245)$ \\
\hline
\end{tabular}

Table 5

Weighted normalized grey decision matrix for aluminium alloys

\begin{tabular}{lccccc}
\hline Aluminium alloy & YS & TS & EF & SED & QI \\
\hline A357FS & $(0.0737,0.0867)$ & $(0.0671,0.0805)$ & $(0.0616,0.0888)$ & $(0.0809,0.1066)$ & $(0.0189,0.0334)$ \\
A357RS & $(0.0741,0.0873)$ & $(0.0653,0.0783)$ & $(0.0400,0.0577)$ & $(0.0516,0.0680)$ & $(0.0175,0.0311)$ \\
A357FC & $(0.0741,0.0873)$ & $(0.0613,0.0735)$ & $(0.0109,0.0157)$ & $(0.0142,0.0187)$ & $(0.0137,0.0244)$ \\
A357RC & $(0.0702,0.0827)$ & $(0.0575,0.0690)$ & $(0.0069,0.0010)$ & $(0.0086,0.0113)$ & $(0.0120,0.0212)$ \\
A224FS & $(0.0625,0.7357)$ & $(0.0698,0.0837)$ & $(0.0397,0.0572)$ & $(0.0540,0.0711)$ & $(0.0184,0.0326)$ \\
A224RS & $(0.0574,0.0676)$ & $(0.0665,0.0798)$ & $(0.0453,0.0653)$ & $(0.0587,0.0774)$ & $(0.0180,0.0320)$ \\
7475FS & $(0.1164,0.1371)$ & $(0.0912,0.1095)$ & $(0.0249,0.0358)$ & $(0.0492,0.0648)$ & $(0.0215,0.0381)$ \\
7475RS & $(0.1130,0.1331)$ & $(0.0885,0.1062)$ & $(0.0132,0.0190)$ & $(0.0254,0.0334)$ & $(0.0195,0.0346)$ \\
\hline
\end{tabular}

Table 6

Ranking of aluminium alloys

\begin{tabular}{lcccccc}
\hline Aluminium alloy & $T^{\max }$ & $T^{\min }$ & $D^{+}$ & $D^{-}$ & $C$ & Rank \\
\hline A357FS & 0.0152 & 0.0152 & 0.087226 & 0.087158 & 0.499805 & 6 \\
A357RS & 0.0074 & 0.0118 & 0.060779 & 0.076708 & 0.557926 & 5 \\
A357FC & 0.0090 & 0.0187 & 0.066897 & 0.096702 & 0.591092 & 2 \\
A357RC & 0.0100 & 0.0222 & 0.070623 & 0.105352 & 0.598677 & 1 \\
A224FS & 0.0076 & 0.0135 & 0.061628 & 0.082257 & 0.571688 & 4 \\
A224RS & 0.0080 & 0.0158 & 0.063069 & 0.089015 & 0.585302 & 3 \\
7475FS & 0.0198 & 0.0041 & 0.099441 & 0.045398 & 0.313437 & 8 \\
7475RS & 0.0177 & 0.0089 & 0.094198 & 0.066526 & 0.413915 & 7 \\
\hline
\end{tabular}


Table 7

Effect of change in greyness on ranking of aluminium alloys

\begin{tabular}{lcccccc}
\hline Aluminium alloy & $1 \%$ & $2 \%$ & $3 \%$ & $5 \%$ & $10 \%$ & Rank \\
\hline A357FS & 0.499805 & 0.499850 & 0.499894 & 0.499981 & 0.500189 & 6 \\
A357RS & 0.557926 & 0.557848 & 0.557771 & 0.557618 & 0.557254 & 5 \\
A357FC & 0.591092 & 0.590842 & 0.590594 & 0.590109 & 0.588952 & 2 \\
A357RC & 0.598677 & 0.598402 & 0.59813 & 0.597597 & 0.596326 & 1 \\
A224FS & 0.571688 & 0.571562 & 0.571438 & 0.571193 & 0.570608 & 4 \\
A224RS & 0.585302 & 0.585189 & 0.585076 & 0.584855 & 0.584327 & 3 \\
7475FS & 0.313437 & 0.313614 & 0.313789 & 0.314134 & 0.314957 & 8 \\
7475RS & 0.413915 & 0.413989 & 0.414062 & 0.414206 & 0.414551 & 7 \\
\hline
\end{tabular}

\subsection{Copper alloys}

Copper and its alloys have the most versatile applications as engineering materials. Some of its favorable properties, like toughness, ductility and malleability make it extremely suitable for tube forming, wire drawing, spinning and deep drawing operations. Other important properties exhibited by copper and its alloys are presented as below:

a) It has high thermal and electrical conductivity, even greater than any other metal except silver.

b) It has good corrosion resistance, which favors durability and long term cost effectiveness.

c) It shows good bio-fouling resistance, as it resists marine organism growth.

d) It has good machinability property, and can be easily machined at the optimal feeds and speeds with proper tools and fixtures.

e) It possesses some favorable mechanical properties, which are often better than those of quenched and tempered steel.

f) It can retain its mechanical and electrical properties at cryogenic temperatures.

g) It has low friction and wear rates. High-leaded tin-bronzes, which are cast into sleeve bearings often have smaller wear rates than steel.

h) All copper alloys can be sand cast and many can be centrifugal, continuous, permanent mold and diecast due to their good castability property.

i) Satistactory surface finish and high tolerance control can be readily achieved due to ease of post-casting processing.

j) Depending upon design loads and corrosivity of the environment, several copper alloys may be the suitable alternative choices for any given industrial application.

k) It has low cost as compared to other metals due to high yield, less machining cost and minimum requirement for surface coating.

With variations in composition and manufacturing methods, these properties of copper and its alloys can be further enhanced. They can be easily cast, have been successfully used over a long period of time and can be readily available from multitude sources. They have a favorable range of physical and mechanical properties, and are quite suitable for machining, brazing, soldering, polishing or plating operation. Cast copper alloys are quite versatile materials. They are being successfully used in plumbing fixtures, ship propellers, power plant water impellers, and bushing and bearing sleeves. In this example, the machinability performances of eight copper alloys, i.e. CuBe1.7 (Be 1.60-1.80\%, Ni + Co 0.20-0.60\%, Cu rest), CuBe2 (Be 1.80-2.10\%, Ni + Co 0.20-0.60\%, Cu rest), CuBe2Pb (Be 1.852.10\%, $\mathrm{Ni}+\mathrm{Co}+\mathrm{Fe}$ 0.20-0.60\%, Pb 0.2-0.6\%, Cu rest), CuCo2Be (Be 0.4-0.7\%, Co 2.4-2.7\%, Cu rest), CuCr1 (Cr 0.4-1.2\%, Cu rest), CuCr1Zr (Cr 0.5-1.5\%, Zr 0.05-0.25\%, Cu rest), CuNiP (Ni 0.470.53\%, P 0.090-0.115\%, Cu rest) and CuNi2Si (Ni 1.6-2.2\%, Si 0.4-0.8\%, Fe $\leq 0.1 \%, \mathrm{Zn} \leq 0.50 \%, \mathrm{Cu}$ rest) are evaluated with respect to five mechanical properties, such as proof strength (in $\mathrm{MPa}$ ), tensile strength (in $\mathrm{MPa}$ ), elongation (in \%), hardness (in BHN) and thermal conductivity (W/m-K). In metallurgy, hardness is the ability of a specific material to resist plastic deformation. Thermal 
conductivity of a material is the quantity of heat transmitted due to unit temperature rise in unit time under steady state. Higher values of proof strength, tensile strength and thermal conductivity are always desired. On the other hand, elongation at fracture and hardness being non-beneficial criteria require their lower values. The grey decision matrix for the eight considered copper alloys is developed as presented in Table 8. In Table 9, the grey weights for various criteria are calculated which are subsequently employed for determining the relative closeness values. From Table 10, it is observed that $\mathrm{CuCr} 1 \mathrm{Zr}$ is the best machinable copper alloy with respect to the considered mechanical properties. Among the eight copper alloys, $\mathrm{CuBe} 2$ is identified as the most difficult one to machine. Although it has the desirable values of proof strength, tensile strength and elongation at fracture, but its high hardness and low thermal conductivity compel it to take the last position in the ranking list. It is also found from Table 10 that the change in greyness in the mechanical properties of copper alloys has no effect on the final rankings of the candidate alloys.

Table 8

Grey decision matrix for copper alloys

\begin{tabular}{lccccc}
\hline Copper alloy & $\begin{array}{c}\text { Proof strength } \\
(\mathrm{PS})\end{array}$ & $\begin{array}{c}\text { Tensile strength } \\
(\mathrm{TS})\end{array}$ & $\begin{array}{c}\text { Elongation at } \\
\text { fracture (EF) }\end{array}$ & Hardness (H) & $\begin{array}{c}\text { Thermal } \\
\text { conductivity (TC) }\end{array}$ \\
\hline CuBe1.7 & $(643.5,656.5)$ & $(841.5,858.5)$ & $(34.95,35.65)$ & $(247.5,252.5)$ & $(29.7,30.3)$ \\
CuBe2 & $(742.5,757.5)$ & $(891,909)$ & $(20,20.4)$ & $(257.4,262.6)$ & $(29.7,30.3)$ \\
CuBe2Pb & $(742.5,757.5)$ & $(891,909)$ & $(20.2,20.6)$ & $(153.45,156.55)$ & $(44.55,45.45)$ \\
CuCo2Be & $(514.8,525.2)$ & $(514.8,525.2)$ & $(25.05,25.55)$ & $(158.4,161.6)$ & $(44.55,45.45)$ \\
CuCr1 & $(267.3,272.7)$ & $(356.4,363.6)$ & $(30.5,31.1)$ & $(123.75,126.25)$ & $(79.2,80.8)$ \\
CuCr1Zr & $(267.3,272.7)$ & $(376.2,383.8)$ & $(35.15,35.85)$ & $(113.85,116.15)$ & $(74.25,75.75)$ \\
CuNiP & $(425.7,434.3)$ & $(519.75,530.25)$ & $(30.2,30.8)$ & $(158.4,161.6)$ & $(49.5,50.5)$ \\
CuNi2Si & $(356.4,363.6)$ & $(495,505)$ & $(35.15,35.85)$ & $(148.5,151.5)$ & $(39.6,40.4)$ \\
\hline
\end{tabular}

Table 9

Grey weights for mechanical properties of copper alloys

\begin{tabular}{lccccc}
\hline Criteria & PS & TS & EF & H & TC \\
\hline Grey weight & $(0.346,0.447)$ & $(0.648,0.748)$ & $(0.245,0.346)$ & $(0.141,0.245)$ & $(0.573,0.674)$ \\
\hline
\end{tabular}

Table 10

Effect of change in greyness on ranking of copper alloys

\begin{tabular}{lcccccc}
\hline Copper alloy & $1 \%$ & $2 \%$ & $3 \%$ & $5 \%$ & $10 \%$ & Rank \\
\hline CuBe1.7 & 0.209138 & 0.209226 & 0.209310 & 0.209910 & 0.209892 & 6 \\
CuBe2 & 0 & 0 & 0 & 0 & 0 & 8 \\
CuBe2Pb & 0.219310 & 0.219479 & 0.219553 & 0.219951 & 0.220771 & 7 \\
CuCo2Be & 0.561381 & 0.561323 & 0.561264 & 0.561185 & 0.560880 & 5 \\
CuCr1 & 0.946531 & 0.946453 & 0.946496 & 0.946226 & 0.945841 & 2 \\
CuCr1Zr & 0.970654 & 0.970612 & 0.970570 & 0.970491 & 0.970289 & 1 \\
CuNiP & 0.664228 & 0.664140 & 0.664053 & 0.663927 & 0.663471 & 4 \\
CuNi2Si & 0.739131 & 0.738893 & 0.738656 & 0.738235 & 0.737077 & 3 \\
\hline
\end{tabular}

\subsection{Alloy steels}

Alloy steel is a type of steel with a variety of alloying elements in total amounts ranging between $1.0 \%$ and $50 \%$ by weight to improve its certain mechanical properties. In alloy steel, carbon is the common alloying element. Apart from carbon, it also contains other major alloyants, like manganese, nickel, chromium, molybdenum, vanadium, silicon and boron. Aluminum, cobalt, copper, cerium, niobium, titanium, tungsten, tin, zinc, lead and zirconium are also added in it in less proportion. Alloy steels can be divided into two main groups, i.e. low alloy steels and high alloy steels. Alloy steel usually refers to low alloy steels. Low alloy steels have better hardenability, which in turn, influences its other mechanical properties. They have increased corrosion resistance in certain environmental conditions. 
Low alloy steel with medium to high carbon content is difficult to weld. When the carbon content is reduced to a range of $0.10 \%$ to $0.30 \%$, along with some change in other alloying elements, the weldability and formability of steel can be substantially increasd while maintaining its strength. This type of steel is known as high strength low alloy steel. Alloy steels may also be divided into four classes, i.e. a) structural steels, which are subjected to stresses in machine parts, b) tool and die steels, c) magnetic alloys, and d) stainless and heat-resisting steels.

When chromium, molybdenum, nickel, manganese and silicon are added, hardness, corrosion resistance, temperature and material strength can be maximized in the basic mix of iron and carbon. Addition of each material must be carefully controlled if the desired result needs to be achieved. From household utensils to buildings to modern art, steel alloys, especially stainless steel have become ubiquitous materials. This alloy has many useful attributes, like bright shine, corrosion and rust-free surface, and durability under harsh weather conditions. Depending on contents of various alloying elements, its specific properties can be enhanced. Alloy steels have wide uses in exotic and highlydemanding applications, like turbine blades in jet engines, landing gear of aircraft and in nuclear reactors. Because of iron's ferromagnetic properties, some steel alloys find important applications in electric motors and transformers where their responses to magnetism are very important.

While selecting the best machinable steel alloy employing grey TOPSIS method, eight candidate alternatives, i.e. AISI 4130 (Fe 97.03-98.22\%, Cr 0.80-1.10\%, Mn 0.40-0.60\%, C 0.28-0.33\%, Si 0.150.30\%, Mo 0.15-0.25\%, S 0.04\%, P 0.035\%), AISI 4340 (Fe 95.195-96.33\%, Ni 1.65-2.99\%, Cr 0.70.9\%, Mn 0.6-0.8\%, C 0.37-0.43\%, Si 0.15-0.30\%, Mo 0.2-0.3\%, S 0.04\%, P 0.035\%), AISI 5140 (Fe 97.395-98.07\%, Cr 0.7-0.9\%, Mn 0.7-0.9\%, C 0.38-0.43\%, Si 0.15-0.30\%, S $\leq 0.04 \%$, P $\leq 0.035 \%$ ), AISI 6150 (Fe 97.095-97.72\%, Cr 0.80-1.10\%, Mn 0.7-0.9\%, C 0.48-0.53\%, Si 0.15-0.30\%, V $\geq$ $0.15 \%, \mathrm{~S} \leq 0.04 \%, \mathrm{P} \leq 0.035 \%$ ), AISI 8650 (Fe 96.54-97.67\%, Cr 0.4-0.6\%, Mn 0.75-1.00\%, C 0.480.53\%, Ni 0.4-0.7\%, Si 0.15-0.30\%, Mo 0.15-0.25\%, S $\leq 0.04 \%, \mathrm{P} \leq 0.035 \%$ ), AISI 8620 (Fe 96.89598.02\%, Cr 0.4-0.6\%, Mn 0.7-0.9\%, C 0.18-0.23\%, Ni 0.4-0.7\%, Si 0.15-0.35\%, Mo 0.15-0.25\%, S $\leq$ $0.04 \%, \mathrm{P} \leq 0.035 \%$ ), AISI 4150 (Fe 96.745-97.67\%, Cr 0.80-1.10\%, Mn 0.75-1.00\%, C 0.48-0.53\%, Si $0.15-0.30 \%$, Mo 0.15-0.25\%, $\mathrm{S} \leq 0.04 \%, \mathrm{P} \leq 0.035 \%$ ) and AISI 8740 (Fe 96.595-97.72\%, Cr 0.40.6\%, Mn 0.75-1.00\%, C 0.38-0.43\%, Ni 0.4-0.7\%, Si 0.15-0.30\%, Mo 0.2-0.3\%, S 0.04\%, P 0.035\%) are considered whose performances are evaluated with respect to five mechanical properties, such as tensile strength (in $\mathrm{MPa}$ ), yield strength (in $\mathrm{MPa}$ ), elastic modulus (in $\mathrm{GPa}$ ), elongation at fracture (in $\%)$ and hardness (in BHN). Among these, tensile strength, yield strength and elastic modulus are beneficial criteria, and the remaining two are non-beneficial in nature. Table 11 exhibits the mechanical properties of the considered alloy steels expressed in grey numbers. The grey weights as calculated for the five mechanical properties of alloy steels are given in Table 12. It is found from Table 13 that AISI 5140 outperforms the other alternatives from the machinability characteristic point of view. With respect to all the five mechanical properties, its performance is satisfactory. AISI 4340 is the most difficult alloy to machine. AISI 5140 has a machinability index of 65 as compared to 50 of AISI 4340. Again, it is observed that the rankings of the alloy steels remain unaltered with the variations in greyness of the considered mechanical properties of the alloys.

Table 11

Grey decision matrix for alloy steels

\begin{tabular}{lccccc}
\hline Alloy steel & $\begin{array}{c}\text { Tensile strength } \\
(\mathrm{TS})\end{array}$ & $\begin{array}{c}\text { Yield strength } \\
(\text { YS })\end{array}$ & $\begin{array}{c}\text { Elastic modulus } \\
(\mathrm{EM})\end{array}$ & $\begin{array}{c}\text { Elongation at } \\
\text { fracture (EF) }\end{array}$ & Hardness (H) \\
\hline AISI 4130 & $(554.4,565.6)$ & $(455.4,464.6)$ & $(198,202)$ & $(15.84,16.16)$ & $(214.83,219.17)$ \\
AISI 4340 & $(737.55,752.45)$ & $(465.3,474.7)$ & $(207.9,212.1)$ & $(21.78,22.22)$ & $(214.83,219.17)$ \\
AISI 5140 & $(564.3,575.7)$ & $(292.05,297.95)$ & $(188.1,191.9)$ & $(19.8,20.2)$ & $(165.33,168.67)$ \\
AISI 6150 & $(663.3,676.7)$ & $(410.85,419.15)$ & $(217.8,222.2)$ & $(22.77,23.23)$ & $(195.03,198.97)$ \\
AISI 8650 & $(707.85,722.15)$ & $(381.15,388.85)$ & $(188.1,191.9)$ & $(22.27,22.72)$ & $(209.88,214.12)$ \\
AISI 8620 & $(524.7,535.3)$ & $(381.15,388.85)$ & $(193.05,196.95)$ & $(24.75,25.25)$ & $(147.51,150.49)$ \\
AISI 4150 & $(723.69,738.31)$ & $(376.2,383.8)$ & $(212.85,217.15)$ & $(19.99,20.40)$ & $(195.03,198.97)$ \\
AISI 8740 & $(688.05,701.95)$ & $(410.85,419.15)$ & $(207.9,212.1)$ & $(21.98,22.42)$ & $(198.99,203.01)$ \\
\hline
\end{tabular}


Table 12

Grey weights for alloy steels

\begin{tabular}{lccccc}
\hline Criteria & TS & YS & EM & EF & H \\
\hline Grey weight & $(0.648,0.748)$ & $(0.573,0.674)$ & $(0.141,0.245)$ & $(0.245,0.346)$ & $(0.346,0.447)$ \\
\hline
\end{tabular}

Table 13

Effect of change in greyness on ranking of alloy steels

\begin{tabular}{lcccccc}
\hline Alloy steel & $1 \%$ & $2 \%$ & $3 \%$ & $5 \%$ & $10 \%$ & Rank \\
\hline AISI 4130 & 0.385168 & 0.385028 & 0.384888 & 0.384613 & 0.383950 & 3 \\
AISI 4340 & 0.239423 & 0.239581 & 0.239737 & 0.240044 & 0.240782 & 8 \\
AISI 5140 & 0.751607 & 0.751447 & 0.751288 & 0.750976 & 0.750225 & 1 \\
AISI 6150 & 0.424285 & 0.424401 & 0.424516 & 0.424742 & 0.425288 & 4 \\
AISI 8650 & 0.413256 & 0.413306 & 0.413357 & 0.413455 & 0.413693 & 5 \\
AISI 8620 & 0.689647 & 0.689689 & 0.689732 & 0.689815 & 0.690015 & 2 \\
AISI 4150 & 0.379471 & 0.379526 & 0.379581 & 0.379689 & 0.379948 & 6 \\
AISI 8740 & 0.369715 & 0.369823 & 0.369929 & 0.370140 & 0.370646 & 7 \\
\hline
\end{tabular}

\section{Conclusions}

In this paper, the machinability characteristics of some alloys of three different metals are studied while employing grey TOPSIS method. The adopted methodology helps the manufacturers to identify the most easily machinable alloys from a list of considered alternatives so that proper machining conditions can be set beforehand. The grey TOPSIS method is found to be quite suitable for this type selection and evaluation problem where the criteria (mechanical properties) values are expressed in grey numbers. It is easily comprehensible and applicable under conflicting decision-making environment. The derived results are observed to be in good agreement with the opinions of the metallurgists and machining professionals. It is also found that the variation in greyness of the mechanical properties of the alloys has no influence on the ranking performance of the adopted methodology. It can be applied to study the machinability characteristics of other alloys too.

\section{References}

Alexopoulos, N.D. \& Pantelakis, S.G. (2004). A new quality index for characterizing aluminum cast alloys with regard to aircraft structure design requirements. Metallurgical and Materials Transactions A, 35A, 301-308.

Alexopoulous, N.D. (2007). Generation of quality maps to support material selection by exploiting the quality indices concept of cast aluminium alloys. Materials \& Design, 28(2), 534-543.

Boubekri, N., Rodriguez, J., \& Asfour, S. (2003). Development of an aggregate indicator to assess the machinability of steels. Journal of Materials Processing Technology, 134(2), 159-165.

Davim, J.P., \& Reis, P. (2004). Machinability study on composite (polyetheretherketone reinforced with 30\% glass fibre-PEEK GF 30) using polycrystalline diamond (PCD) and cemented carbide (K20) tools. International Journal of Advanced Manufacturing Technology, 23(5-6), 412-418.

Davim, J. P., \& Mata, F. (2005). A new machinability index in turning fiber reinforced plastics. Journal of Materials Processing Technology, 170(1-2), 436-440.

Davim, J.P., \& Figueira, L. (2007). Machinability evaluation in hard turning of cold work tool steel (D2) with ceramic tools using statistical techniques. Materials \& Design, 28(4), 1186-1191.

Deng, J. L. (1982). Control problem of grey system. System and Control Letters, 1(5), 288-294.

Deng, J. L. (1989). Introduction to grey system theory. The Journal of Grey System, 1(1), 1-24.

Enache, S., Străjescu, E., Opran, C., Minciu, C., \& Zamfirache, M. (1995). Mathematical model for the establishment of the materials machinability. Annals of the CIRP, 44(1), 79-82. 
Hoseiny, H., Högman, B., Klement, U., \& Kinnander, A. (2012). Machinability evaluation of prehardened plastic mould steels. International Journal of Machining and Machinability of Materials, 11(4), 327-341.

Jadidi, O., Hong, T.S., Firouzi, F., \& Yusuff, R.M. (2008). An optimal grey based approach based on TOPSIS concepts for supplier selection problem. International Journal of Management Science and Engineering Management, 4(2), 104-117.

Kim, K-K., Kang, M-C., Kim, J-S., Jung, Y-H., \& Kim, N-K. (2002). A study on the precision machinability of ball end milling by cutting speed optimization. Journal of Materials Processing Technology, 130-131, 357-362.

Li, G-D., Yamaguchi, D., \& Nagai, M. (2007). A grey-based decision-making approach to the supplier selection problem. Mathematical and Computer Modelling, 46(3-4), 573-581.

Lin, Y-H., Lee, P-C., \& Ting, H-I. (2008). Dynamic multi-attribute decision making model with grey number evaluations. Expert Systems with Applications, 35(4), 1638-1644.

Lobato, F.S., Sousa, M.N., Silva, M.A.. \& Machado, A.R. (2014). Multi-objective optimization and bio-inspired methods applied to machinability of stainless steel. Applied Soft Computing, 22, 261271.

Manna, A., \& Bhattacharayya, B. (2003). A study on machinability of Al/SiC-MMC. Journal of Materials Processing Technology, 140(1-3), 711-716.

Mills, B. (1983). Machinability of Engineering Materials. Essex: Applied Science Publishers Ltd.

Morehead, M., Huang, Y., \& Hartwig, K.T. (2007). Machinability of ultrafine-grained copper using tungsten carbide and polycrystalline diamond tools. International Journal of Machine Tools \& Manufacture, 47(2), 286-293.

Rao, R.V., \& Gandhi, O.P. (2002). Digraph and matrix methods for the machinability evaluation of work materials. International Journal of Machine Tools \& Manufacture, 42(3), 321-330.

Rao, R.V. (2006). Machinability evaluation of work materials using a combined multiple attribute decision-making method. International Journal of Advanced Manufacturing Technology, 28(3-4), 221-227.

Rech, J., Le Calvez, C., \& Dessoly, M. (2004). A new approach for the characterization of machinability - application to steels for plastic injection molds. Journal of Materials Processing Technology, 152(1), 66-70.

Sadeghi, M., Razavi, S.H., \& Saberi, N. (2013). Application of grey TOPSIS in preference ordering of action plans in balanced scorecard and strategy map. Informatica, 24(4), 619-635.

Šalak, A., Vasilko, K., Selecká, M., \& H. Danninger (2006). New short time face turning method for testing the machinability of PM steels. Journal of Materials Processing Technology, 176(1-3), 6269.

Sameer Kumar, D., \& Suman, K.N.S. (2014). Selection of magnesium alloy by MADM methods for automobile wheels. International Journal of Engineering and Manufacturing, 4(2), 31-41.

Şeker, U., \& Hasirci, H. (2006). Evaluation of machinability of austempered ductile irons in terms of cutting forces and surface quality. Journal of Materials Processing Technology, 173(3), 260-268.

Sridharan, V., \& Muthukrishnan, N. (2013). Optimization of machinability of polyester/modified jute fabric composite using grey relational analysis (GRA). Procedia Engineering, 64, 1003- 1012.

Stoić, A., Kopač, J., \& Cukor, G. (2005). Testing of machinability of mould steel 40CrMnMo7 using genetic algorithm. Journal of Materials Processing Technology, 164-165, 1624-1630.

Turskis, Z., \& Zavadskas, E.K. (2010). A novel method for multiple criteria analysis: grey additive ratio assessment (ARAS-G) method. Informatica, 21(4), 597-610.

Wang, X., Huang, C., Zou, B., Liu, H., Zhu, H., \& Wang, J. (2014). A new method to evaluate the machinability of difficult-to-cut materials. International Journal of Advanced Manufacturing Technology, 75(1-4), 91-96.

Xu, L., Schultheiss, F., Andersson, M., \& Ståhl, J-E. (2013). General conception of polar diagrams for the evaluation of the potential machinability of workpiece materials. International Journal of Machining and Machinability of Materials, 14(1), 24-44. 
Zavadskas, E.K., Kaklauskas, A., Turskis, Z., \& Tamošaitienė, J. (2009). Multi-attribute decisionmaking model by applying grey numbers. Informatica, 20(2), 305-320.

Zolfani, S.H., \& Antucheviciene, J. (2012). Team member selecting based on AHP and TOPSIS grey. Inzinerine Ekonomika - Engineering Economics, 23(4), 425-434. 